\title{
Zooplankton diversity of three floodplain lakes (Beels) of the Majuli River Island, Brahmaputra River Basin of Assam, Northeast India
}

\begin{abstract}
The biodiverse zooplankton of Bhereki, Holmari and Ghotonga beels of Majuli River Island, the Brahmaputra river basin of upper Assam, northeast India (NEI) revealed total richness of $141(118 \pm 8)$ species and thus suggested habitat diversity of these floodplain wetlands. Low community similarities, monthly richness variations and the cluster groupings affirmed heterogeneity of zooplankton species composition. Zooplankton formed the dominant component of net plankton in Ghotonga beel and showed sub-dominance in Bhereki and Holmari beels. Rotifera $>$ Rhizopoda influenced zooplankton density in Bhereki and Ghotonga beels; Rhizopoda > Rotifera showed importance in Holmari beel; and Copepoda $>$ Cladocera recorded sub-dominance in all beels. Zooplankton is characterized by higher species diversity and equitability, and lower dominance. The richness, abundance and diversity of zooplankton and abundance of the constituent groups followed oscillating monthly variations. While explaining limited influence of individual abiotic factors and low cumulative influence along two axes (vide Canonical Correspondence Analysis), our results suggested that zooplankton are largely generalists in terms of abiotic factors and thus hypothesized importance of factors associated with microhabitat.
\end{abstract}

Keywords: Aluvial floodplains, Abundance, Composition, Density, Richness, Tropical Wetlands
Volume 6 Issue I - 2017

BK Sharma, MK Hatimuria

Department of Zoology, North-Eastern Hill University, India

Correspondence: BK Sharma, Freshwater Biology, Department of Zoology, North-Eastern Hill University, India, Email profbksharma@gmail.com

Received: June 12, 2017 | Published: July 18, 2017

\section{Introduction}

The floodplain lakes form an integral component of various riverine systems globally and merit interest for biodiversity and ecology considerations. These remarks hold valid for the Indian floodplains and those of northeast India (NEI) in particular. ${ }^{1-3}$ These wetlands form a lucrative source of inland fishery ${ }^{4}$ of NEI and are locally known as 'beels' in Assam and pats in Manipur. Further, zooplankton contribute importantly to metazoan diversity and production of fish-food organisms in the wetlands but have yet received inadequate attention on their diversity and ecology in the floodplain lakes of India ${ }^{1}$ while the related published ecology works from NEI are limited to reports from certain beels of Assam ${ }^{5-7}$ and pats of Manipur. ${ }^{8,9}$

Majuli, a geologically interesting landform of fluvial geomorphology of the Brahmaputra river basin of upper Assam as well as a world heritage site, is under threat of extinction because of alarming erosion. This largest riverine island is literally dotted with beels with vital socio-economic importance due to notable fisheries potential. Our knowledge of plankton communities in the floodplains of Majuli River Island is limited to the faunal diversity of Rotifera ${ }^{10,11}$ and Cladocera. ${ }^{12}$ This first study on zooplankton diversity of selected Majuli beels merits ecological and aquaculture interest in the Indian floodplain lakes in general and those of NEI in particular; referring to the latter aspect, these floodplains presently yield 500$700 \mathrm{~kg} \mathrm{fish} / \mathrm{ha} / \mathrm{yr}$ which can be significantly enhanced 3-4 times through scientific management ${ }^{4}$ based on knowledge of diversity and production of fish-food organisms. The observations are made on monthly variations of richness and abundance of zooplankton and its constituent groups vis-a-vis individual and cumulative influence of abiotic factors; and community similarities, species diversity and evenness and dominance.

\section{Materials and methods}

This limnological survey was undertaken during September, 2010-August, 2012 in Bhereki beel (94o08'23.3" E, 26055'40.4" N; Altitude: $72 \mathrm{~m} \mathrm{ASL})$, Holmari beel (94o12'30.6"E; 26059'17.3"N) and Ghotonga beel (94o15'28.7"E, 27o01'52.7"N; Altitude: $69 \mathrm{~m}$ ASL) located in Majuli River Island in the Jorhat district of Upper Assam (N. E. India). The sampled wetlands indicated a diversity of macrophytes namely Eichhornia crassipes, Hydrilla verticellata, Utricularia flexuosa, Trapa bispinosa, Lemna major, L. minor, Pistia striates, Salvinia sp., Nymphaea spp., Nymphoides spp., Vallisneria spiralis, Euryale ferox, Xanthium sp., Ipomoea fistulosa \& Sagittaria sp.

Water samples were collected at regular monthly intervals and analyzed for various abiotic factors. Water temperature, specific conductivity and $\mathrm{pH}$ were recorded by field probes, dissolved oxygen was estimated by the modified Winkler's method and other parameters were analyzed following APHA. ${ }^{13}$ Qualitative zooplankton samples were collected from the floodplain lakes by towing any lobolt plankton net (\# $50 \mu \mathrm{m}$ ) and preserved in 5\% formalin. These samples were subsequently screened for various zooplankton species and their permanent mounts were made in polyvinyl alcohol-Lactophenol mixture.

Monthly quantitative zooplankton samples were also obtained by filtering 25 litres of the lake water through nylobolt plankton net (No. 25). Individual collections were then concentrated to $25 \mathrm{ml}$ each and preserved in $5 \%$ formalin. The quantitative enumeration $(\mathrm{n} / \mathrm{l})$ was done with the help of a Sedgewick-Rafter counting cell. The zooplankton was identified following the works of. ${ }^{1,14-19}$ Quantitative samples were analyzed for abundance of zooplankton. Community similarity (Sørensen's index) and species diversity (Shannon's index) were calculated following. ${ }^{20,21}$ ANOVA was used to analyze 
the significance of temporal variation of the biotic communities. Ecological relationships between abiotic and biotic parameters of Bhereki beel, Holmari beel and Ghotonga beel were determined by simple correlation co-efficient $\left(\mathrm{r}_{1}, \mathrm{r}_{2}\right.$ and $\mathrm{r}_{3}$, respectively); $\mathrm{P}$ values were calculated and their significance was ascertained after the use of Bonferroni correction. The canonical correspondence analysis (XLSTAT 2014) was done to analyse cumulative influence of seventeen abiotic parameters (water temperature, rainfall, $\mathrm{pH}$, specific conductivity, dissolved oxygen, free $\mathrm{CO}_{2}$, total alkalinity, total hardness, calcium, magnesium, chloride, dissolved organic matter, total dissolved solids, phosphate, nitrate, sulphate and silicate) on the zooplankton assemblages.

\section{Results}

The ranges and mean $\pm \mathrm{SD}$ of the recorded abiotic parameters of
Bhereki, Holmari and Ghotonga beels are indicated in Table 1 and of different aspects of zooplankton diversity are included in Table 2. We observed a total of 141 with 111, 113 and 129 species in three beels, respectively (Table 2). Rotifera is represented by 70, 66 and 79 species in Bhereki, Holmari and Ghotonga beels, respectively (Table 3) and indicated qualitative importance of Lecanidae > Lepadellidae $>$ Brachionidae. The monthly zooplankton richness varied between 45 64, 47-67 and 49-76 species (Figures 1-3); it recorded 48.8-78.3\%, $49.1-74.3 \%$ and $48.7-69.7 \%$ community similarities (vide Sørensen's index) in three beels, respectively. The hierarchical cluster analysis of zooplankton is presented in Figures 4-9. The monthly rotifer richness ranged between $21-35,22-39$ and $23-45$ species while that of Cladocera (33 species) varied between $5-14,4-14$ and 8-18 species the sampled beels, respectively.

Table I Abiotic factors of Majuli beels (September 20 I0-August 20I2)

\begin{tabular}{|c|c|c|c|c|c|c|}
\hline \multirow{2}{*}{ Factors } & \multicolumn{2}{|l|}{ Bhereki beel } & \multicolumn{2}{|l|}{ Holmari beel } & \multicolumn{2}{|l|}{ Ghotonga beel } \\
\hline & Range & Mean \pm Sd & Range & Mean \pm Sd & Range & Mean \pm Sd \\
\hline Water temperature $(\mathrm{oC})$ & $21.5-27.5$ & $23.7 \pm 1.7$ & $21.0-27.5$ & $23.6 \pm 1.7$ & $21.5-27.5$ & $23.9 \pm 1.7$ \\
\hline Rainfall (mm) & $0.0-413.76$ & $142.57 \pm 133.90$ & $0.0-413.76$ & $142.57 \pm 133.90$ & $0.0-413.76$ & $142.57 \pm 133.90$ \\
\hline $\mathrm{pH}$ & $6.29-7.41$ & $6.67 \pm 0.23$ & $6.56-7.13$ & $6.87 \pm 0.13$ & $6.17-6.85$ & $6.51 \pm 0.16$ \\
\hline Conductivity $(\mu \mathrm{S} / \mathrm{cm})$ & $102.0-189.0$ & $140.7 \pm 24.4$ & III.0-220.0 & $173.6 \pm 32.5$ & $73.0-182.0$ & $121.4 \pm 26.8$ \\
\hline Dissolbed oxygen (mg/l) & $4.8-8.0$ & $6.3 \pm 0.9$ & $5.6-8.0$ & $7.1 \pm 0.8$ & $4.0-8.0$ & $6.2 \pm 1.0$ \\
\hline Free $\mathrm{CO}_{2}(\mathrm{mg} / \mathrm{l})$ & $6.0-24.0$ & $13.6 \pm 4.0$ & $6.0-16.0$ & $10.2 \pm 2.8$ & $6.0-20.0$ & $13.8 \pm 3.4$ \\
\hline Alkalinity (mgl-I) & $44.0-126.0$ & $70.3 \pm 20.7$ & $64.0-116.0$ & $92.3 \pm 14.2$ & $38.0-88.0$ & $62.2 \pm 13.4$ \\
\hline Hardness (mg/l) & $42.0-128.0$ & $69.8 \pm 20.3$ & $56.0-122.0$ & $89.3 \pm 16.9$ & $38.0-84.0$ & $60.8 \pm 13.6$ \\
\hline Calcium (mg/l) & $27.3-81.9$ & $43.0 \pm 13.1$ & $37.8-73.5$ & $60.2 \pm 9.2$ & $25.2-54.6$ & $38.7 \pm 7.8$ \\
\hline Magnesium (mg/l) & $1.34-11.91$ & $6.51 \pm 2.81$ & $2.19-11.88$ & $7.08 \pm 2.41$ & $1.02-11.30$ & $5.38 \pm 2.34$ \\
\hline Chloride (mg/l) & $5.99-32.97$ & $10.99 \pm 5.25$ & $3.99-21.98$ & $8.91 \pm 3.49$ & $6.99-39.96$ & $13.15 \pm 6.54$ \\
\hline DOM (mg/l) & $0.041-0.319$ & $0.162 \pm 0.062$ & $0.026-0.278$ & $0.113 \pm 0.047$ & $0.038-0.353$ & $0.166 \pm 0.063$ \\
\hline TDS (mg/l) & $0.088-0.172$ & $0.137 \pm 0.023$ & $0.080-0.160$ & $0.115 \pm 0.022$ & $0.104-0.180$ & $0.147 \pm 0.020$ \\
\hline Phosphate (mg/l) & $0.145-3.619$ & $0.963 \pm 0.697$ & $0.093-1.582$ & $0.76 \mathrm{I} \pm 0.393$ & $0.165-1.499$ & $0.845 \pm 0.414$ \\
\hline Nitrate $(\mathrm{mg} / \mathrm{l})$ & $0.501-4.522$ & $1.855 \pm 1.047$ & $0.544-4.411$ & $1.800 \pm 1.030$ & $0.499-3.566$ & $1.758 \pm 0.838$ \\
\hline Sulphate (mg/l) & $1.387-17.776$ & $8.789 \pm 4.161$ & $0.793-14.075$ & $6.473 \pm 3.741$ & $0.925-13.282$ & $7.219 \pm 3.600$ \\
\hline Silicate $(\mathrm{mg} / \mathrm{l})$ & $0.140-2.652$ & $0.880 \pm 0.547$ & $0.140-2.547$ & $0.825 \pm 0.511$ & $0.140-1.187$ & $0.660 \pm 0.275$ \\
\hline
\end{tabular}

Table 2 Species composition of zooplankton of Majuli beels

\begin{tabular}{|c|c|c|c|}
\hline $\begin{array}{l}\text { Taxa } \downarrow \\
\text { Beels } \rightarrow\end{array}$ & Bhereki & Ghotonga & Holmari \\
\hline \multicolumn{4}{|l|}{$\begin{array}{l}\text { Phylum: Rotifera } \\
\text { Subclass: Monogononta }\end{array}$} \\
\hline \multicolumn{4}{|l|}{ Order: Ploima } \\
\hline \multicolumn{4}{|l|}{ Family:Asplanchnidae } \\
\hline I. Asplanchna priodonta Gosse & + & + & + \\
\hline \multicolumn{4}{|l|}{ Family: Brachionidae } \\
\hline 2. Anuraeopsis fissa Gosse & + & + & - \\
\hline Brachionus angularis Gosse & - & - & - \\
\hline Brachionus durgae Dhanapathi & - & - & - \\
\hline Brachionus falcatus Zacharias & - & - & - \\
\hline Brachionus kostei Shiel & - & - & - \\
\hline Brachionus quadridentatus Hermann & + & + & + \\
\hline Keratella cochlearis (Gosse) & + & + & + \\
\hline 9. Keratella edmondsoni Ahlstrom & - & - & - \\
\hline 10. Keratella lenzi Hauer & + & + & - \\
\hline II. Keratella tecta (Gosse) & - & + & - \\
\hline 12. Keratella tropica (Apstein) & + & - & + \\
\hline 13. Platyias quadricornis (Ehrenberg) & + & + & + \\
\hline I4. Plationus patulus (O.F. Muller) & + & + & + \\
\hline \multicolumn{4}{|l|}{ Family: Euchlanidae } \\
\hline 15. Beauchampiella eudactylota (Gosse) & + & + & + \\
\hline Dipleuchlanis propatula (Gosse) & + & - & + \\
\hline Euchlanis dilatata Ehrenberg & + & + & + \\
\hline Euchlanis triquetra Ehrenberg & + & + & - \\
\hline 19. Tripleuchlanis plicata (Levander) & - & + & + \\
\hline
\end{tabular}


Table Continued...

\begin{tabular}{|c|c|c|c|c|}
\hline \multicolumn{2}{|c|}{$\begin{array}{l}\text { Taxa } \downarrow \\
\text { Beels } \rightarrow\end{array}$} & Bhereki & Ghotonga & Holmari \\
\hline \multicolumn{5}{|c|}{ Family: Flosculariidae } \\
\hline 20. & Sinantherina socialis (Linne) & - & + & - \\
\hline & Sinantherina spinosa (Thorpe) & + & + & + \\
\hline \multicolumn{5}{|c|}{ Family: Lecanidae } \\
\hline 22. & Lecane aculeata (Jakubski) & + & + & + \\
\hline 23. & Lecane arcula Harring & - & - & - \\
\hline 24. & Lecane bifurca (Bryce) & - & - & - \\
\hline 25. & Lecane blachei Berzins & - & + & + \\
\hline 26. & Lecane bulla (Gosse) & + & + & + \\
\hline 27. & Lecane closterocerca (Schmarda) & + & + & + \\
\hline 28. & Lecane crepida Harring & - & + & + \\
\hline 29. & Lecane curvicornis (Murray) & + & + & - \\
\hline 30. & Lecane decipiens (Murray) & - & - & - \\
\hline 31. & Lecane doryssa Harring & + & + & - \\
\hline 32. & Lecane flexilis (Gosse) & - & - & - \\
\hline 33. & Lecane furcata (Murray) & + & + & + \\
\hline 34. & Lecane haliclysta Harring \& Myers & - & - & - \\
\hline 35. & Lecane hamata (Stokes) & + & + & + \\
\hline 36. & Lecane hornemanni (Ehrenberg) & + & + & + \\
\hline 37. & Lecane inermis (Bryce) & + & + & + \\
\hline 38. & Lecane inopinata Harring \& Myers & + & + & - \\
\hline 39. & Lecane lateralis Sharma & + & + & + \\
\hline 40. & Lecane leontina (Turner) & + & + & + \\
\hline 41. & Lecane ludwigii (Eckstein) & + & + & + \\
\hline 42. & Lecane luna (O.F. Müller) & + & + & + \\
\hline 43. & Lecane lunaris (Ehrenberg) & + & + & + \\
\hline 44. & Lecane monostyla (Daday) & - & + & - \\
\hline 45. & Lecane nitida (Murray) & - & + & + \\
\hline 46. & Lecane niwati Segers, Kotethip \& Sanoamuang & - & - & - \\
\hline 47. & Lecane obtusa (Murray) & + & + & + \\
\hline 48. & Lecane ohioensis (Herrick) & + & + & - \\
\hline 49. & Lecane papuana (Murray) & + & + & + \\
\hline 50. & Lecane paxiana Hauer & - & - & - \\
\hline $5 \mathrm{I}$. & Lecane ploenensis (Voigt) & + & + & + \\
\hline 52. & Lecane pusilla Harring & - & + & + \\
\hline 53. & Lecane pyriformis (Daday) & + & + & + \\
\hline 54. & Lecane quadridentata (Ehrenberg) & + & + & + \\
\hline 55. & Lecane rhytida Harring \& Myers & - & - & - \\
\hline 56. & Lecane signifera (Jennings) & + & + & + \\
\hline 57. & Lecane stenroosi (Meissner) & + & + & + \\
\hline 58. & Lecane styrax (Harring \& Myers) & - & - & - \\
\hline 59. & Lecane tenuiseta Harring & - & - & - \\
\hline 60. & Lecane thienemanni (Hauer) & - & - & - \\
\hline 61. & Lecane undulata Hauer & - & + & - \\
\hline 62. & Lecane unguitata (Fadeev) & + & + & + \\
\hline 63. & Lecane ungulata (Gosse) & + & + & + \\
\hline \multicolumn{5}{|c|}{ Family: Lepadellidae } \\
\hline 64. & Colurella adriatica Ehrenberg & - & - & - \\
\hline 65. & Colurella obtusa (Gosse) & + & + & + \\
\hline 66. & Colurella uncinata (O.F. Müller) & + & + & + \\
\hline 67. & Lepadella acuminata(Ehrenberg) & + & + & + \\
\hline 68. & Lepadella apsida Harring & + & + & - \\
\hline 69. & Lepadella benjamini Harring & + & + & + \\
\hline 70. & Lepadella biloba Hauer & - & - & - \\
\hline 7I. & Lepadella costatoides Segers & - & - & - \\
\hline 72. & Lepadella dactyliseta (Stenroos) & - & - & - \\
\hline 73. & Lepadella discoidea Segers & + & + & + \\
\hline 74. & Lepadella elongata Koste & + & + & - \\
\hline 75. & Lepadella lindaui Koste & - & - & - \\
\hline 76. & Lepadella minuta (Weber \& Montet) & - & - & - \\
\hline 77. & Lepadella ovalis (O.F. Muller) & + & + & + \\
\hline 78. & Lepadella patella (O.F. Muller) & + & + & + \\
\hline 79. & Lepadella quinquecostata (Lucks) & - & - & - \\
\hline 80. & Lepadella rhomboides (Gosse) & + & + & + \\
\hline 81. & Lepadella triba Myers & - & - & - \\
\hline
\end{tabular}


Table Continued..

\begin{tabular}{|c|c|c|c|c|}
\hline \multicolumn{2}{|c|}{$\begin{array}{l}\text { Taxa } \downarrow \\
\text { Beels } \rightarrow\end{array}$} & \multirow{2}{*}{$\begin{array}{l}\text { Bhereki } \\
-\end{array}$} & \multirow{2}{*}{$\begin{array}{l}\text { Ghotonga } \\
-\end{array}$} & \multirow{2}{*}{$\begin{array}{l}\text { Holmari } \\
-\end{array}$} \\
\hline 82. & Lepadella triptera Ehrenberg & & & \\
\hline 83. & Lepadella vandenbrandei Gillard & - & - & - \\
\hline 84. & Lepadella (Heterolepadella) apsicora Myers & - & - & - \\
\hline 85. & Lepadella (H.) ehrenbergi Perty & + & + & + \\
\hline 86. & Lepadella (H.) heterostyla (Murray) & - & + & - \\
\hline \multicolumn{5}{|c|}{ Family: Mytilinidae } \\
\hline 87. & Lophocharis oxysternon (Gosse) & - & - & + \\
\hline & Mytilina acanthophora Hauer & + & + & - \\
\hline 89. & Mytilina bisulcata (Lucks) & + & - & + \\
\hline & Mytilina michelangellii Reid \& Turner & - & - & - \\
\hline & Mytilina ventralis (Ehrenberg) & + & + & + \\
\hline \multicolumn{5}{|c|}{ Family: Notommatidae } \\
\hline 92. & Cephalodella forficula (Ehrenberg) & - & + & + \\
\hline 93. & Cephalodella gibba ( Ehrenberg) & + & + & + \\
\hline 94. & Monommata longiseta (O.F. Müller) & + & + & + \\
\hline \multicolumn{5}{|c|}{ Family: Scaridiidae } \\
\hline 95. & Scaridium longicaudum ( Müller) & + & + & + \\
\hline \multicolumn{5}{|c|}{ Family: Synchaetidae } \\
\hline 96. & Pleosoma lenticulare Herrick & - & - & - \\
\hline 97. & Polyarthra vulgaris Carlin & + & + & + \\
\hline \multicolumn{5}{|c|}{ Order: Flosculariaceae } \\
\hline \multicolumn{5}{|c|}{ Family: Conochilidae } \\
\hline & Conochilus unicornis Rousselet & - & - & - \\
\hline \multicolumn{5}{|c|}{ Family: Hexarthridae } \\
\hline & Hexarthra mira (Hudson) & - & - & - \\
\hline \multicolumn{5}{|c|}{ Family:Testudinellidae } \\
\hline 100. & Testudinella amphora Hauer & - & - & - \\
\hline 101. & Testudinella emarginula Stenroos & + & + & + \\
\hline 102. & Testudinella patina (Hermann) & + & + & + \\
\hline 103. & Testudinella tridentata Smirnov & - & - & - \\
\hline 104. & Pompholyx sulcata Hudson & + & + & + \\
\hline \multicolumn{5}{|c|}{ Family:Trichocercidae } \\
\hline 105. & Trichocerca bicristata (Gosse) & - & - & - \\
\hline 106. & Trichocerca cylindrica (Imhof) & + & + & + \\
\hline 107. & Trichocerca elongata (Gosse) & - & - & - \\
\hline 108. & Trichocerca insignis (Herrick) & + & + & + \\
\hline 109. & Trichocerca rattus (O.F. Muller) & + & + & + \\
\hline 110. & Trichocerca scipio (Gosse) & - & - & - \\
\hline III. & Trichocerca similis (Wierzejski) & + & + & + \\
\hline 112. & Trichocerca tigris (O.F. Muller) & + & + & - \\
\hline 113. & Trichocerca uncinata (Voigt) & - & - & - \\
\hline 114. & Trichocerca weberi (Jennings) & - & - & - \\
\hline \multicolumn{5}{|c|}{ Family:Trichotriidae } \\
\hline 115. & Macrochaetus longipes Myers & - & + & + \\
\hline 116. & Macrochaetus sericus (Thorpe) & + & + & + \\
\hline 117. & Trichotria tetractis (Ehrenberg) & + & + & + \\
\hline Famil & ly:Trochosphaeridae & & & \\
\hline 118. & Filinia camasecla Myers & - & - & - \\
\hline 119. & Filinia longiseta (Ehrenberg) & + & + & + \\
\hline 120. & Trochosphaera aequatorialis Semper & - & - & - \\
\hline Sub-c & lass: Digononta & & & \\
\hline Orde & r: Bdelloidea & & & \\
\hline Famil & y: Philodinidae & & & \\
\hline 121. & Philodina citrina Ehrenberg & + & + & + \\
\hline 122. & Rotaria neptunia (Ehrenberg) & + & + & - \\
\hline 123. & Rotaria rotatoria (Pallas) & - & - & + \\
\hline Super- & order: Cladocera (sensu strictu) & & & \\
\hline Family & : Bosminidae & & & \\
\hline 124. & Bosmina longirostris Sars s.lat & - & + & - \\
\hline 125. & Bosminopsis deitersi Richard & - & - & - \\
\hline Famil & y: Chydoridae & & & \\
\hline Subfa & mily:Aloninae & & & \\
\hline 126. & Alona affinis (Leydig) s.lat & - & - & - \\
\hline 127. & Alona cheni Sinev & - & + & - \\
\hline 128. & Alona guttata tuberculata Kurz & + & - & + \\
\hline
\end{tabular}

Citation: Sharma BK, Hatimuria MK. Zooplankton diversity of three floodplain lakes (Beels) of the Majuli River Island, Brahmaputra River Basin of Assam, Northeast India. J Aquac Mar Biol. 2017;6(I):I I-12. DOI: 10.15406/jamb.2017.06.00I44 
Table Continued...

\begin{tabular}{|c|c|c|c|c|}
\hline \multicolumn{2}{|l|}{$\begin{array}{l}\text { Taxa } \downarrow \\
\text { Beels } \rightarrow\end{array}$} & \multirow{2}{*}{$\begin{array}{l}\text { Bhereki } \\
-\end{array}$} & \multirow{2}{*}{$\begin{array}{l}\text { Ghotonga } \\
-\end{array}$} & \multirow{2}{*}{ Holmari } \\
\hline 129. & Alona kotovi Sinev & & & \\
\hline 130 & Anthalona harti Van Damme et al. & - & - & - \\
\hline$|3|$. & Camptocercus uncinatus Smirnov & + & + & - \\
\hline 132. & Celsinotum macronyx (Daday) & - & + & - \\
\hline 133. & Celsinotum rectangula (Sars) s.lat & + & - & + \\
\hline 134. & Euryalona orientalis (Daday) & - & + & + \\
\hline 135. & Graptoleberis testudinaria (Fischer) & - & + & + \\
\hline 136. & Karualona karua (King) & + & + & + \\
\hline 137. & Kurzia brevilabris Rajapaksa \& Fernando & - & - & - \\
\hline 138. & Kurzia latissima Kurz & - & + & + \\
\hline 139. & Kurzia longirostris (Daday) & + & + & - \\
\hline 140. & Leberis diphanus (King) & - & - & - \\
\hline |4I. & Notoalona globulosa (Daday) & + & + & + \\
\hline 142. & Oxyurella singalensis (Daday) & - & - & - \\
\hline \multicolumn{5}{|c|}{ Subfamily: Chydorinae } \\
\hline 143. & Alonella clathratula Sars & - & + & + \\
\hline 144. & Alonella excisa (Fischer) & + & + & + \\
\hline 145. & Chydorus angustirostris Frey & + & + & - \\
\hline 146. & Chydorus sphaericus (O. F. Muller) & + & + & + \\
\hline 147. & Chydorus ventricosus Daday & - & + & + \\
\hline 148. & Dadaya macrops (Daday) & - & - & - \\
\hline 149. & Disperalona caudata Smirnov & + & - & + \\
\hline 150. & Dunhevedia crassa King & - & - & - \\
\hline$|5|$. & Dunhevedia serrata Daday & + & + & - \\
\hline 152. & Picripleuroxus quasidenticulatus (Smirnov) & - & - & - \\
\hline 153. & Picripleuroxus similis Vavra & + & + & + \\
\hline \multicolumn{5}{|c|}{ Family: Daphniidae } \\
\hline 154. & Ceriodaphnia cornuta Sars & + & + & + \\
\hline 155. & Scapholeberis kingi Sars & + & + & - \\
\hline 156. & Simocephalus acutirostratus(King) & - & - & + \\
\hline 157. & Simocephalus serrulatus (Koch) & + & + & + \\
\hline I58. & Simocephalus mixtus Sars & + & + & - \\
\hline \multicolumn{5}{|c|}{ Family: Ilyocryptidae } \\
\hline 159. & Ilyocryptus spinifer Herrick & - & - & - \\
\hline \multicolumn{5}{|c|}{ Family: Macrothricidae } \\
\hline 160. & Grimaldina brazzai Richard & - & - & - \\
\hline 161. & Guernella raphaelis Richard & - & - & + \\
\hline 162. & Macrothrix laticornis (Fischer) & + & + & + \\
\hline 163. & Macrothrix spinosa King & - & - & - \\
\hline 164. & Macrothrix triserialis (Brady) & + & + & + \\
\hline \multicolumn{5}{|c|}{ Family: Moinidae } \\
\hline 165. & Moina micrura Kurz & - & + & + \\
\hline 166. & Moinodaphnia macleayi (King) & - & + & + \\
\hline \multicolumn{5}{|c|}{ Order: Ctenopoda } \\
\hline \multicolumn{5}{|c|}{ Family: Sididae } \\
\hline 167. & Diaphanosoma excisum Sars & + & + & + \\
\hline 168. & Diaphanosoma sarsi Richard & + & - & - \\
\hline 169. & Diaphanosoma senegal Gauthier & - & - & - \\
\hline 170. & Pseudosida szalayi (Daday) & - & - & - \\
\hline I7I. & Sida crystallina (O. F. Muller) & - & + & + \\
\hline \multicolumn{5}{|c|}{ Sub-Kingdom: Protozoa } \\
\hline \multicolumn{5}{|c|}{ Super-class: Rhizopoda } \\
\hline 172. & Arcella discoides Ehrenberg & + & + & + \\
\hline 173. & Arcella hemispherica Perty & + & + & + \\
\hline 174. & Arcella vulgaris Ehrenberg & + & + & + \\
\hline \multicolumn{5}{|c|}{ Family: Centropyxidae } \\
\hline 175. & Centropyxis aculeata (Ehrenberg) & + & + & + \\
\hline 176. & Centropyxis ecornis (Ehrenberg) & + & + & + \\
\hline 177. & Centropyxis oblonga (Deflandre) & + & + & + \\
\hline Family: D & Difflugidae & & & \\
\hline 178. & Difflugia acuminata Ehrenberg & + & + & + \\
\hline 179. & Difflugia corona Wallich & + & + & + \\
\hline 180. & Difflugia oblonga Ehrenberg & + & + & + \\
\hline$|8|$ & Difflugia urceolata Carter & - & + & + \\
\hline Family: E & Euglyphidae & & & \\
\hline
\end{tabular}

Citation: Sharma BK, Hatimuria MK. Zooplankton diversity of three floodplain lakes (Beels) of the Majuli River Island, Brahmaputra River Basin of Assam, Northeast India. J Aquac Mar Biol. 2017;6(I): I I-I2. DOI: 10.15406/jamb.2017.06.00144 
Table Continued...

\begin{tabular}{|c|c|c|c|c|}
\hline \multicolumn{2}{|c|}{$\begin{array}{l}\text { Taxa } \downarrow \\
\text { Beels } \rightarrow\end{array}$} & \multirow{2}{*}{$\begin{array}{l}\text { Bhereki } \\
+\end{array}$} & \multirow{2}{*}{$\begin{array}{l}\text { Ghotonga } \\
+\end{array}$} & \multirow{2}{*}{$\begin{array}{l}\text { Holmari } \\
++\end{array}$} \\
\hline 182. & Assulina muscorum Greet & & & \\
\hline 183. & Euglypha acanthophora Dujardin & + & + & + \\
\hline 184. & E. tuberculata Dujardin & - & + & + \\
\hline 185. & Trinema enchelys (Ehrenberg) & - & - & + \\
\hline \multicolumn{5}{|c|}{ Family: Nebelidae } \\
\hline 186. & Lesquereusia spiralis (Ehrenberg) & + & + & + \\
\hline 187. & Nebela caudata Leidy & + & + & + \\
\hline \multicolumn{5}{|c|}{ Class: Copepoda } \\
\hline \multicolumn{5}{|c|}{ Family: Cyclopidae } \\
\hline 188. & Tropocyclops prasinus (Fischer) & + & + & + \\
\hline 189. & Mesocyclops leuckarti (Claus) & + & + & + \\
\hline 190. & Microcyclops varicans Sars & + & + & + \\
\hline 191. & Thermocyclops decipiens & + & + & + \\
\hline \multicolumn{5}{|c|}{ Family: Diaptomidae } \\
\hline 192. & Heliodiaptomus cinctus & + & + & + \\
\hline 193. & Neodiaptomus schmackeri & + & + & + \\
\hline Class & stracoda & & & \\
\hline \multicolumn{5}{|c|}{ Family: Cyprididae } \\
\hline 194. & Cypris subglobulosa & + & + & + \\
\hline 195. & Strandesia indica & + & + & + \\
\hline 196. & Hemicypris anomala & - & - & - \\
\hline \multicolumn{5}{|c|}{ Species Richness } \\
\hline Rotif & & 70 & 79 & 66 \\
\hline Cladc & & 20 & 27 & 23 \\
\hline Rhizo & & 13 & 15 & 16 \\
\hline Cope & & 6 & 6 & 6 \\
\hline Ostra & & 2 & 2 & 2 \\
\hline Total & lankton & III & 129 & 113 \\
\hline
\end{tabular}

Table 3 Temporal variations of zooplankton (September 2010-August, 2012)

\begin{tabular}{|c|c|c|c|c|c|c|}
\hline beels $\rightarrow$ & \multicolumn{2}{|l|}{ Bhereki beel } & \multicolumn{2}{|l|}{ Holmari beel } & \multicolumn{2}{|c|}{ Ghotonga beel } \\
\hline \multicolumn{7}{|l|}{ Qualitative } \\
\hline Net Plankton & 209 species & & 212 species & & 232 species & \\
\hline Zooplankton & I I I species & & I 3 species & & 129 species & \\
\hline Percentage similarity \% & $48.8-75.7$ & & $49.1-74.3$ & & $46.1-69.7$ & \\
\hline Zooplankton (species) & $45-64$ & $54 \pm 6$ & $47-67$ & $57 \pm 6$ & $49-76$ & $63 \pm 8$ \\
\hline Rotifera & $21-35$ & $28 \pm 4$ & $22-39$ & $28 \pm 4$ & $23-45$ & $33 \pm 6$ \\
\hline Cladocera & $5-14$ & $10 \pm 2$ & $4-14$ & $11 \pm 3$ & $8-18$ & $13 \pm 3$ \\
\hline \multicolumn{7}{|l|}{ Quantitative } \\
\hline Net Plankton $\left(\mathrm{nl}^{-1}\right)$ & $261-1253$ & $663 \pm 261$ & $449-1815$ & $682 \pm 289$ & $282-1923$ & $628 \pm 320$ \\
\hline Zooplankton $\left(\mathrm{nl}^{-1}\right)$ & $173-388$ & $245 \pm 52$ & $163-523$ & $275 \pm 87$ & $187-448$ & $293 \pm 71$ \\
\hline$\%$ composition & $23.8-73.2$ & $40.5 \pm 12.5$ & $\mid 5.2-61.0$ & $42.9 \pm 12.3$ & $15.4-78.0$ & $51.4 \pm 13.5$ \\
\hline Diversity & $3.012-3.793$ & $3.555 \pm 0.184$ & $3.245-4.042$ & $3.650 \pm 0.197$ & $3.464-4.111$ & $3.813 \pm 0.172$ \\
\hline Dominance & $0.063-0.196$ & $0.115 \pm 0.036$ & $0.048-0.174$ & $0.099 \pm 0.031$ & $0.042-0.139$ & $0.082 \pm 0.028$ \\
\hline Evenness & $0.755-0.944$ & $0.893 \pm 0.041$ & $0.803-0.962$ & $0.905 \pm 0.039$ & $0.877-0.970$ & $0.924 \pm 0.032$ \\
\hline Rotifera $\left(\mathrm{nl}^{-1}\right)$ & $44-132$ & $80 \pm 22$ & $48-179$ & $89 \pm 32$ & $6 I-22 I$ & $119 \pm 37$ \\
\hline \% composition & $23.9-52.0$ & $32.8 \pm 6.5$ & || $.9-5 \mid .7$ & $34.0 \pm 10.1$ & $25.8-52.4$ & $40.4 \pm 6.7$ \\
\hline Cladocera $\left(\mathrm{nl}^{-1}\right)$ & $15-99$ & $38 \pm 20$ & $12-59$ & $33 \pm 14$ & $24-116$ & $52 \pm 21$ \\
\hline$\%$ composition & $7.7-28.2$ & $15.3 \pm 6.2$ & $6.5-18.9$ & $11.9 \pm 3.5$ & $10.4-28.8$ & $17.7 \pm 5.1$ \\
\hline Rhizopoda ( $\left.\mathrm{nl}^{-1}\right)$ & $22-133$ & $72 \pm 31$ & $39-254$ & $99 \pm 57$ & $39-|4|$ & $76 \pm 26$ \\
\hline \% composition & $8.4-47.0$ & $29.5 \pm 11.0$ & $16.1-65.2$ & $34.3 \pm 11.6$ & $12.5-37.8$ & $26.4 \pm 7.5$ \\
\hline Copepoda $\left(\mathrm{nl}^{-1}\right)$ & $13-154$ & $52 \pm 32$ & $12-120$ & $50 \pm 26$ & $17-108$ & $42 \pm 23$ \\
\hline$\%$ composition & $4.6-56.4$ & $21.2 \pm 11.9$ & $3.8-43.8$ & $18.4 \pm 8.7$ & $7.5-32.6$ & $\mid 4.1 \pm 6.1$ \\
\hline Ostracoda $\left(\mathrm{nl}^{-1}\right)$ & $0-9$ & $3 \pm 2$ & $1-10$ & $4 \pm 3$ & $0-8$ & $4 \pm 3$ \\
\hline \multicolumn{7}{|l|}{ Important families $\left(\mathrm{nl}^{-1}\right)$} \\
\hline Lecanidae & $24-60$ & $37 \pm 9$ & $18-106$ & $40 \pm 21$ & $12-80$ & $44 \pm 15$ \\
\hline Lepadellidae & $4-23$ & $12 \pm 5$ & $6-21$ & $\mathrm{II} \pm 4$ & $6-30$ & $17 \pm 7$ \\
\hline Brachionidae & $3-32$ & $10 \pm 6$ & $2-19$ & $8 \pm 5$ & $2-64$ & $22 \pm 20$ \\
\hline Chydoridae & $8-65$ & $20 \pm 11$ & $7-35$ & $19 \pm 8$ & $15-84$ & $30 \pm 16$ \\
\hline Arcellidae & $6-77$ & $39 \pm 22$ & $8-104$ & $28 \pm 21$ & $13-53$ & $30 \pm 11$ \\
\hline Centropyxidae & $5-29$ & $11 \pm 6$ & $3-52$ & $23 \pm 15$ & $1-62$ & $16 \pm 4$ \\
\hline Difflugidae & $0-23$ & $10 \pm 7$ & $3-42$ & $14 \pm 9$ & $1-32$ & $12 \pm 8$ \\
\hline
\end{tabular}

Citation: Sharma BK, Hatimuria MK. Zooplankton diversity of three floodplain lakes (Beels) of the Majuli River Island, Brahmaputra River Basin of Assam, Northeast India. J Aquac Mar Biol. 2017;6(I):I I-12. DOI: I0.15406/jamb.2017.06.00I44 
Table Continued...

\begin{tabular}{|c|c|c|c|c|c|c|}
\hline \multirow{2}{*}{$\frac{\text { Taxa } \downarrow}{\text { Euglephidae }}$} & \multicolumn{2}{|c|}{ Bhereki beel } & \multicolumn{2}{|c|}{ Holmari beel } & \multicolumn{2}{|c|}{ Ghotonga beel } \\
\hline & $0-14$ & $5 \pm 4$ & $1-69$ & $18 \pm 16$ & $I-25$ & $9 \pm 7$ \\
\hline Macrothricidae & $0-28$ & $7 \pm 7$ & $0-17$ & $5 \pm 4$ & $0-28$ & $\mathrm{II} \pm 8$ \\
\hline \multicolumn{7}{|l|}{ Important species $\left(\mathrm{nl}^{-1}\right)$} \\
\hline Arcella discoides & $0-36$ & $16 \pm 10$ & $2-40$ & $11 \pm 9$ & $5-28$ & $13 \pm 6$ \\
\hline Tropocyclops prasinus & $4-53$ & $20 \pm 14$ & $2-40$ & $16 \pm 10$ & $3-4 I$ & $14 \pm 10$ \\
\hline A. vulgaris & $0-40$ & $14 \pm 10$ & $2-63$ & $12 \pm 13$ & $4-34$ & $10 \pm 6$ \\
\hline Centropyxis aculeata & $1-15$ & $5 \pm 4$ & $0-30$ & $14 \pm 9$ & $0-40$ & $10 \pm 9$ \\
\hline Euglypha acanthophora & $0-14$ & $4 \pm 4$ & $0-55$ & $10 \pm 12$ & $0-20$ & $6 \pm 5$ \\
\hline Nebela caudata & $1-16$ & $7 \pm 4$ & $2-42$ & $11 \pm 10$ & $3-16$ & $8 \pm 3$ \\
\hline Thermocyclops decipiens & $5-51$ & $17 \pm 11$ & $0-30$ & $6 \pm 7$ & $0-29$ & $8 \pm 7$ \\
\hline Mesocyclops leuckarti & $0-32$ & $9 \pm 7$ & $2-34$ & $16 \pm 9$ & $3-30$ & $10 \pm 8$ \\
\hline Microcyclops varicans & $4-18$ & $5 \pm 4$ & $\mathrm{I}-34$ & $10 \pm 7$ & $0-29$ & $8 \pm 7$ \\
\hline Macrothrix triserialis & $0-22$ & $6 \pm 6$ & $0-4$ & $1 \pm 1$ & $0-28$ & $10 \pm 8$ \\
\hline
\end{tabular}

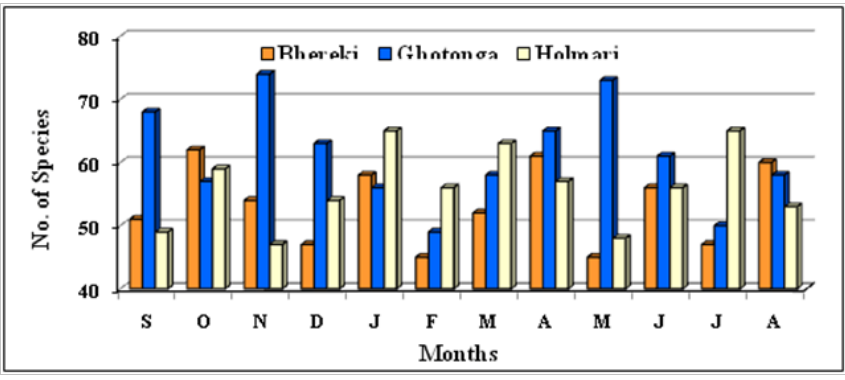

Figure I Monthly variations in Species Richness of Zooplankton (2010 20II).

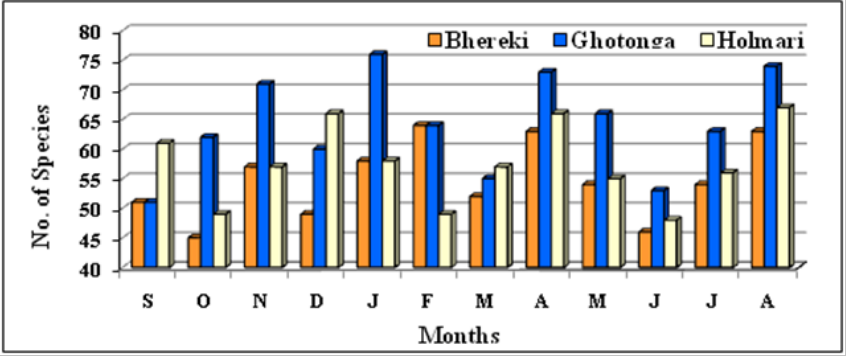

Figure 2 Monthly variations in Species Richness of Zooplankton (201I 2012).

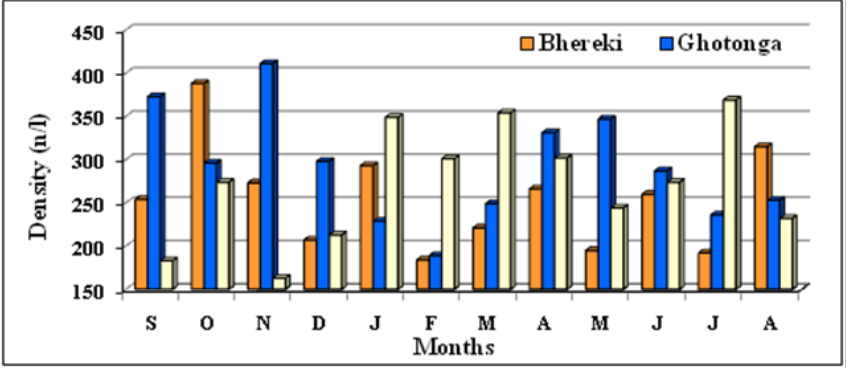

Figure 3 Monthly variations in abundance of Zooplankton (2010 - 20I I).

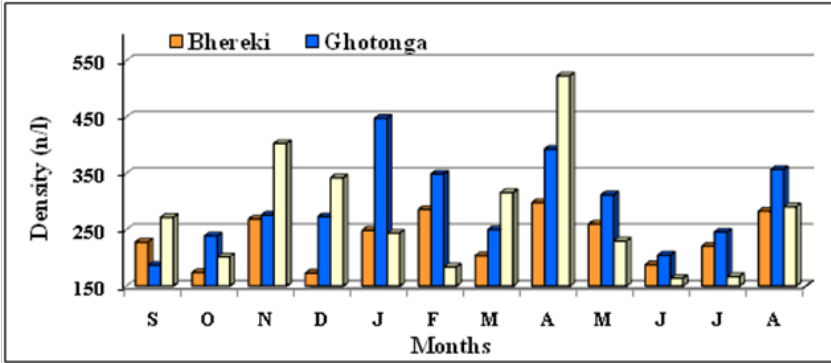

Figure 4 Monthly variations in abundance of Zooplankton (20II - 20I2).

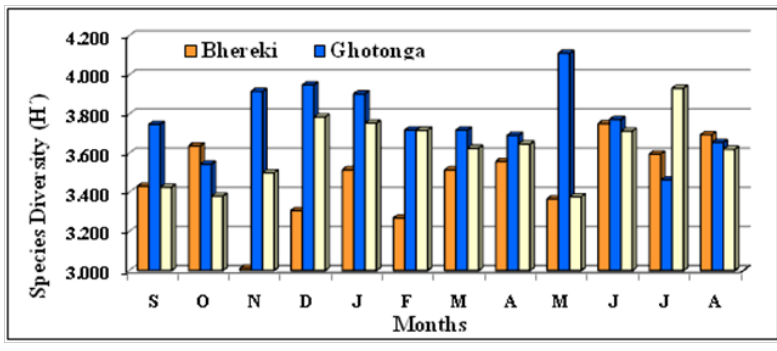

Figure 5 Monthly variations of species diversity of Zooplankton (2010 20II).

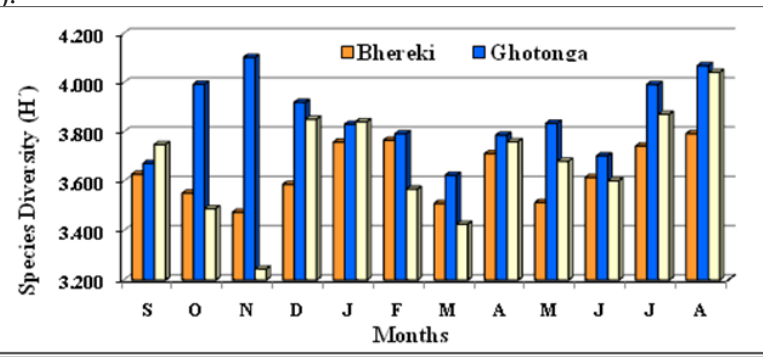

Figure 6 Monthly variations of species diversity of Zooplankton (2011 2012).

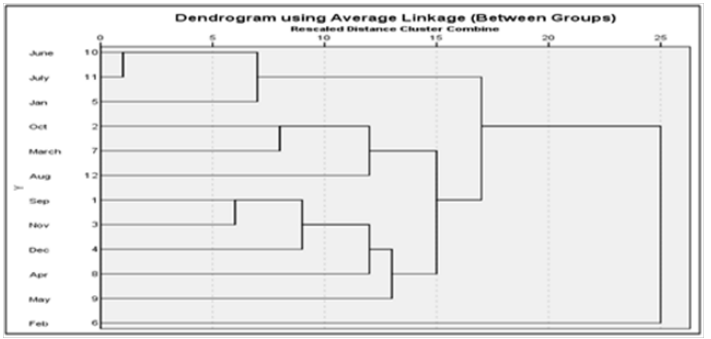

Figure 7 Hierarchical cluster analysis of zooplankton of Bhereki beel $(2010$ $-20 \mathrm{II})$.

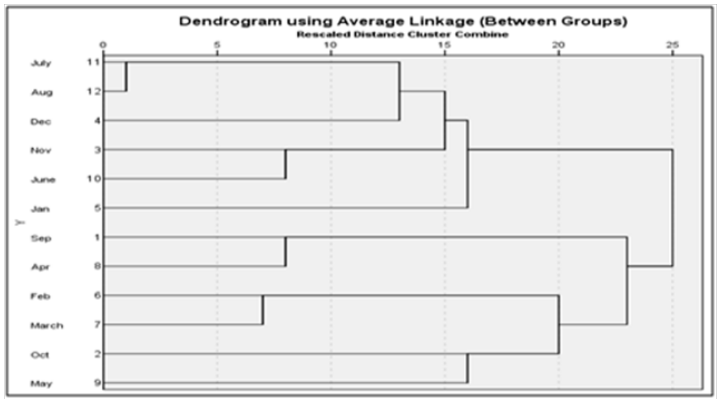

Figure 8 Hierarchical cluster analysis of zooplankton of Bhereki beel (201I - 2012).

Citation: Sharma BK, Hatimuria MK. Zooplankton diversity of three floodplain lakes (Beels) of the Majuli River Island, Brahmaputra River Basin of Assam, Northeast India. J Aquac Mar Biol. 2017;6(I):I I-12. DOI: I0.15406/jamb.2017.06.00I44 


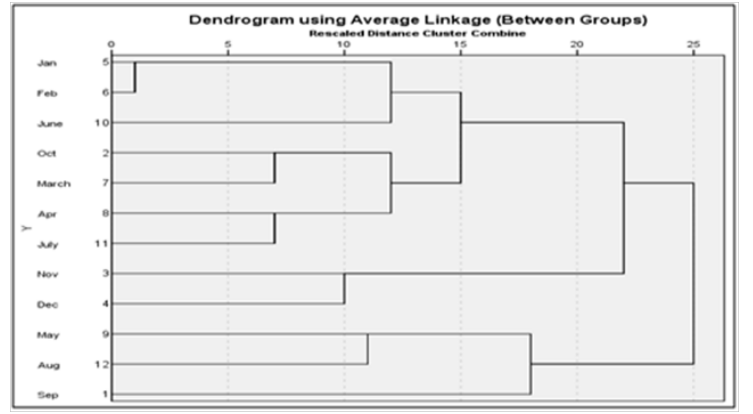

Figure 9 Hierarchical cluster analysis of zooplankton of Holmari beel (2010 - 201I).

Zooplankton density (Table 3 ) ranged between 173-388, 163-523 and $187-448 \mathrm{n} / 1$ (Figures $10 \& 11$ ); it comprised $40.5 \pm 12.5,42.9 \pm 12.3$ and $51.4 \pm 13.5 \%$ of net plankton of Bhereki, Holmari and Ghotonga beels, respectively. Rotifera density varied between 44-132, $(80 \pm 22)$, 48-179 (89 \pm 32$)$ and 61-221 (119 \pm 37$) \mathrm{n} / \mathrm{l}$ and comprised 32.8 \pm 6.5 , $34.0 \pm 10.1$ and $40.4 \pm 6.7 \%$ (Table 2) of zooplankton; Rhizopoda showed density variations between 22-133 (72 \pm 31$), 39-254(99 \pm 57)$

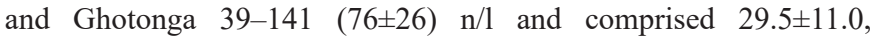
$34.3 \pm 11.6$ and $26.4 \pm 7.5 \%$ of zooplankton; Copepoda density varied between 13-154 (52 \pm 32$), 12-120(50 \pm 26)$ and 17-108 (42 \pm 23$) n / 1$ and comprised between $21.2 \pm 11.9,18.4 \pm 8.7$ and $7.5-14.1 \pm 6.1 \%$ of zooplankton; and Cladocera abundance varied between 15-99 $(38 \pm 20), 12-59(33 \pm 14)$ and $24-116(52 \pm 21) \mathrm{n} / \mathrm{l}$ and formed between $15.3 \pm 6.2,11.9 \pm 3.5$ and $17.7 \pm 5.1 \%$ of zooplankton abundance of three beels, respectively (Table 3 ). The species diversity, dominance and evenness varied (Table 2) between 3.012-3.793, 3.245-4.042 and 3.464-4.111 (Figures $12 \& 13$ ); 0.063-0.196, 0.048-0.174 and 0.042$0.139 ; 0.755-0.944,0.803-0.962$ and $0.877-0.970$ in the sampled beels, respectively. The CCA ordination biplots of zooplankton assemblages and abiotic factors of three beels are indicated in Figures14-16, respectively.

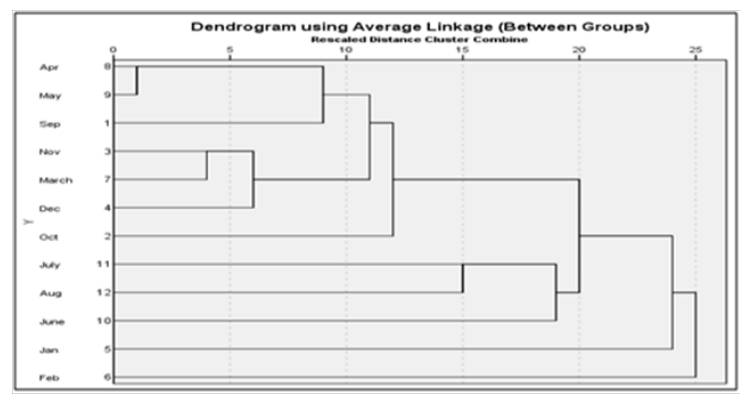

Figure 10 Hierarchical cluster analysis of zooplankton of Holmari beel (201 I - 2012).

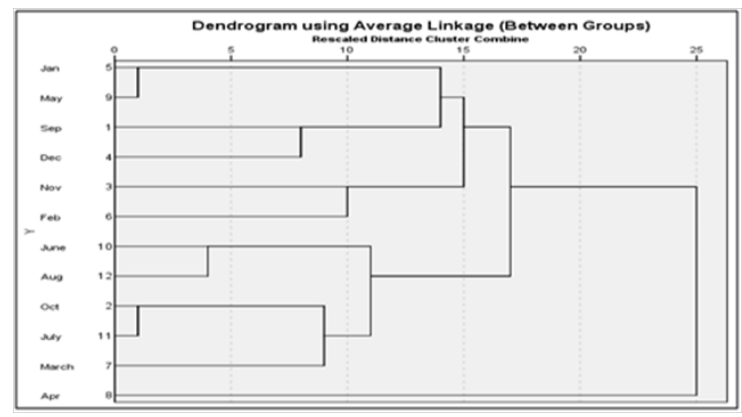

Figure I I Hierarchical cluster analysis of zooplankton of Ghotonga beel (2010 - 20II).

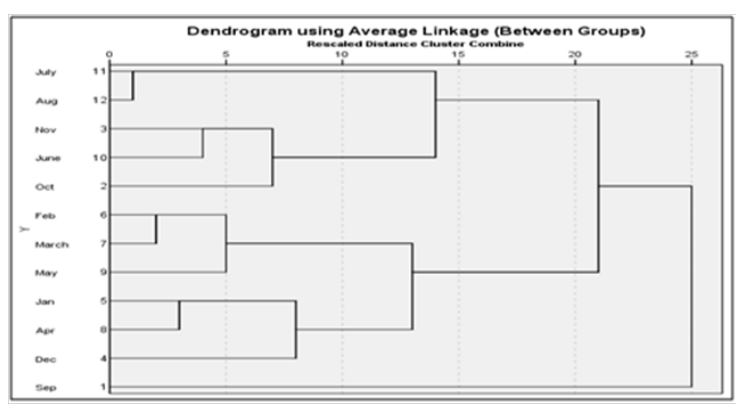

Figure 12 Hierarchical cluster analysis of zooplankton of Ghotonga beel (20II - 20I2).

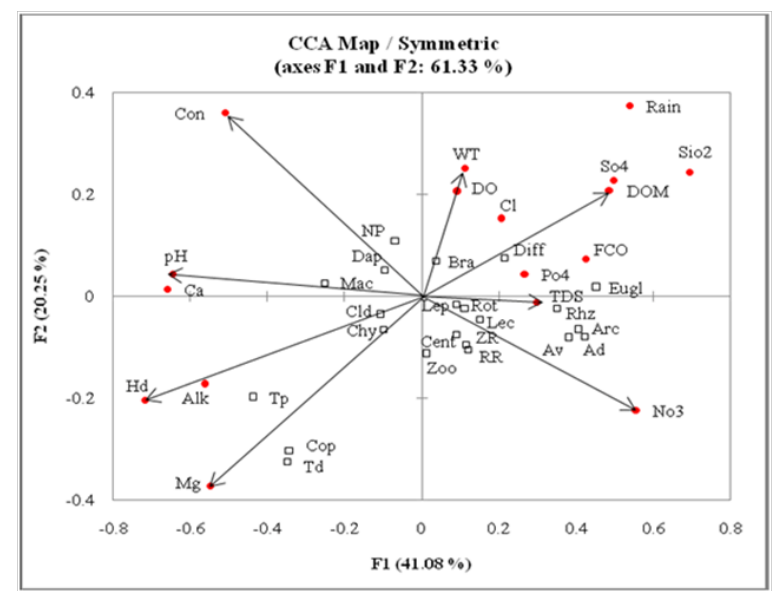

Figure 13 CCA ordination biplot of Zooplankton assemblages and environmental variables (Bhereki beel).

Abiotic: ALK: Alkalinity; Ca: Calcium; Cl: Chloride; CON: Conductivity; DO: Dissolved Oxygen; DOM: Dissolved Oxygen Matter; FCO: Free Carbon Dioxide; HD: Hardness; Mg: Magnesium; pH Hydrogen-lon Concentration; $\mathrm{NO}_{3}$ : Nitrate; $\mathrm{PO}_{4}$ : Phosphate; Rain: Rainfall; $\mathrm{SIO}_{2}$ : Silicate; $\mathrm{SO}_{4}$ : Sulphate;TDS: Total Dissolved Solids; WT:Water Temperature

Biotic: AD: Arcella discoides;ARC:Arcellidae;Av: A. vulgaris; BRA: Brachionidae; CEN: Centropyxidae; CHY: Chydoridae; CLD: Cladocera; COP: Copepoda; DAP: Daphniidae; DIFF: Difflugidae; EUGL: Euglephidae; LEC: Lecanidae; LEP Lepadellidae; MAC: Macrothricidae; NP: Net Plankton; ORHZ: Rhizopoda; ROT: Rotifera; RR: Rotifera Richness; TD: Thermocyclops Decipiens; TP: Tropocyclops Prasinus; ZP: Zooplankton; ZR: Zooplankton Richness.

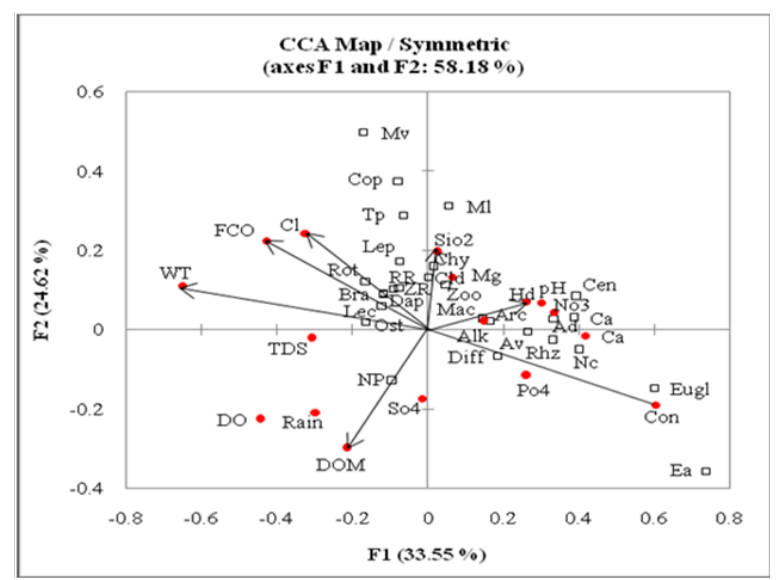

Figure I4 CCA ordination biplot of Zooplankton assemblages and environmental variables (Holmari beel). 
Abbreviations: Abiotic: ALK: Alkalinity; Ca: Calcium; Cl: Chloride; CON: Conductivity; DO: Dissolved oxygen; DOM: Dissolved Oxygen Matter; FCO: Free Carbon Dioxide; HD: Hardness; Mg: Magnesium; $\mathrm{pH}$ Hydrogen-ion concentration; $\mathrm{NO}_{3}$ : Nitrate; $\mathrm{PO}_{4}$ : phosphate; Rain: rainfall; $\mathrm{SIO}_{2}$ : silicate; $\mathrm{SO}_{4}$ : sulphate;TDS:Total dissolved solids;WT: water temperature.

Biotic: AD: Arcella discoides;ARC:Arcellidae;Av: A. vulgaris; BRA: Brachionidae; CEN: Centropyxidae; CHY: Chydoridae; CLD: Cladocera; COP: Copepoda; DAP: Daphniidae; DIFF: Difflugidae; EUGL: Euglephidae; LEC: Lecanidae; LEP Lepadellidae; MAC:Macrothricidae; NP: Net plankton; ORHZ: Rhizopoda; ROT: Rotifera; RR: Rotifera richness; TD: Thermocyclops decipiens; TP: Tropocyclops prasinus; ZP: Zooplankton; ZR: Zooplankton richness.

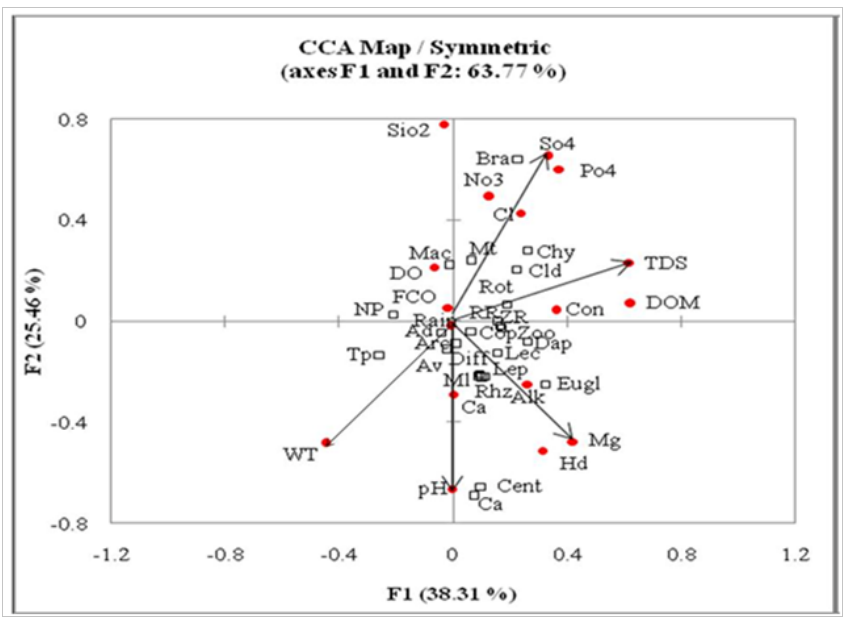

Figure 15 CCA ordination biplot of Zooplankton assemblages and environmental variables (Ghotonga beel).

Abbreviations: Abiotic: ALK: Alkalinity; Ca: Calcium; Cl: Chloride; CON Conductivity; DO: Dissolved oxygen; DOM: Dissolved Oxygen Matter; FCO: Free Carbon Dioxide; HD: Hardness; Mg: Magnesium; pH Hydrogen-ion concentration; $\mathrm{NO}_{3}$ : Nitrate; $\mathrm{PO}_{4}$ : phosphate; Rain: rainfall; $\mathrm{SIO}_{2}$ : silicate; $\mathrm{SO}_{4}$ : sulphate;TDS:Total dissolved solids;WT: water temperature

Biotic: AD: Arcella discoides;ARC:Arcellidae;Av: A. vulgaris; BRA: Brachionidae; CEN: Centropyxidae; CHY: Chydoridae; CLD: Cladocera; COP: Copepoda; DAP: Daphniidae; DIFF: Difflugidae; EUGL: Euglephidae; LEC: Lecanidae; LEP: Lepadellidae; MAC: Macrothricidae; MI: Mesocyclops leuckarti; MT: Macrothrix triserialis; NP: Net plankton; RHZ: Rhizopoda; ROT: Rotifera; RR: Rotifera richness; TP: Tropocyclops prasinus; ZP: Zooplankton; ZR: Zooplankton richness.

\section{Discussion}

\section{Abiotic parameters}

Water temperature concurred with the geographical location of the sampled beels. Bhereki and Holmari beels are characterized by slightly acidic to circum-neutral waters whereas Ghotonga beel indicated slightly acidic waters. The specific conductivity exhibited low ionic concentration of the three beels and warranted of these wetlands under 'Class I' category of trophic classification. ${ }^{22}$ All three floodplain lakes are characterized by moderately hard water character, moderate dissolved oxygen, low free $\mathrm{CO}_{2}$, low chloride content, and relatively low concentrations of dissolved organic matter and total dissolved solids.

\section{Zooplankton richness}

Zooplankton (141 species) of Majuli beels are more biodiverse than the reports from various beels of Assam, ${ }^{1}$ two floodplain lakes of Manipur, ${ }^{8}$ certain lakes of Kashmir Himalayas, ${ }^{23,24}$ two Kumaun lakes of Uttarakhand, ${ }^{25,26}$ two wetlands of Barak river basin of Assam, ${ }^{27}$ two floodplain lakes of southwest Bengal ${ }^{28}$ and from two wetlands of Kashmir. ${ }^{29}$ The zooplankton richness of individual Majuli beels $(118 \pm 8$ species) is yet relatively lower than the report of 143 species enlisted from Ghorajan beel of Assam ${ }^{7}$ it is reasonably comparable with 123 species known from a sub-tropical lake of Jammu province ${ }^{30}$ while it is higher than 93 species known from Dal Lake ${ }^{31}$ and 85 species from Wular wetland ${ }^{32}$ of Kashmir; and 70 species from a floodplain wetland of West Bengal. ${ }^{33}$

Zooplankton contributed significantly to net plankton richness in Bhereki $\left(r_{1}=0.732, p<0.0001\right)$, Holmari $\left(r_{2}=0.705, p=0.0001\right)$ and Ghotonga $\left(\mathrm{r}_{3}=0.749, \mathrm{p}<0.0001\right)$ beels. The monthly richness followed broadly concurrent variations in Bhereki ( $54 \pm 6$ species) and Holmari ( $57 \pm 6$ species) beels than marginally high richness in Ghotonga beel (63 \pm 8 species). ANOVA indicated significant richness variations amongst three beels $\left(\mathrm{F}_{2,71}=11.201, \mathrm{P}=0.0001\right)$. The community similarities (vide Sørensen's index) of 48.8-75.7\%, 49.1-77.6\% and $46.1-69.7 \%$ in Bhereki, Holmari and Ghotonga beels, respectively coupled with monthly richness variations suggested heterogeneity in zooplankton composition in individual beels. The hierarchical cluster analysis of Bhereki beel indicated high zooplankton affinities between June vs. July during the first year; and between July vs. August during second year while peak divergence is noticed during February and September $>$ February $>$ October during two years, respectively. In Holmari beel, maximum affinity was recorded between January vs. February and April vs. May collections while November $>$ September $>$ May and February $>$ January $>$ June communities indicated high divergence during two years of the study period, respectively. Further in Ghotonga beel, high affinities are indicated between January vs. May and again between October vs. July communities during first year and between July vs. August communities during second year while maximum divergence is noted during April and September collections during two years, respectively. The cluster groupings indicated distinct annual variations, during two years, in three beels individually and thus affirmed heterogeneity in monthly composition of zooplankton communities.

The richness followed oscillating temporal variations in the sampled beels concurrent with the reports of Sharma and Sharma ${ }^{7}$ and Sharma ${ }^{6,8}$ while it differed from winter and autumn maxima reported from Loktak Lake, Manipur. ${ }^{9}$ Peak richness of 64, 67 and 76 species was observed during winter (February, 2012), monsoon (August, 2012) and winter (January, 2012) in Bhereki, Holmari and Ghotonga beels, respectively. Rotifera ( 84 species), the most species-rich group of Ghotonga $>$ Bhereki $>$ Holmari beels contributed significantly to temporal variations of zooplankton richness $\left(\mathrm{r}_{1}=0.884, \mathrm{p}<0.0001\right.$; $\left.\mathrm{r}_{2}=0.661, \mathrm{p}=0.0003 ; \mathrm{r}_{3}=0.875, \mathrm{p}<0.0001\right)$. Besides, Cladocera contributed significantly to zooplankton richness in the sampled beels $\left(r_{1}=0.736, p<0.0001 ; r_{2}=0.782, p<0.0001 ; r_{3}=0.804, p<\right.$ $0.0001)$. More remarks on the diversity of the stated groups are made separately. ${ }^{11,12}$

Zooplankton abundance was relatively higher in Ghotonga beel $(293 \pm 71 \mathrm{n} / \mathrm{l})$ than that of Bhereki $(245 \pm 52 \mathrm{n} / \mathrm{l})$ and Holmari $(275 \pm 87$ $\mathrm{n} / \mathrm{l}$ ) beels; it registered insignificant annual as well as monthly density variations amongst the three beels as well as in the individual beels. The recorded zooplankton abundance concurred with the reports from Ghorajan beel of Assam, ${ }^{7}$ and two floodplain lakes of Manipur. ${ }^{8,9}$ Further, the recorded densities are lower than the reports from Surinsar lake of Kashmir, ${ }^{30}$ Deepor Beel - a Ramsar site ${ }^{6}$ of Assam, and the results from various Indian floodplain lakes. ${ }^{28,} 29$. ${ }^{34-36}$ Zooplankton followed oscillating patterns of monthly density variations with peaks during post-monsoon (October, 2010), summer (April, 2012) and winter (January, 2012) in Bhereki, Holmari and Ghotonga beels, respectively. The winter peak of Ghotonga beel concurred with reports of Sharma ${ }^{6-8}$ and Sharma and Sharma ${ }^{9}$ while 
oscillating patterns agreed with the report of Sharma and Sharma ${ }^{12}$ but differed from bimodal pattern noted by Sanjer and Sharma ${ }^{35}$ but. Zooplankton formed the dominant quantitative component of net plankton in Ghotonga beel $(51.4 \pm 13.5 \%)$ while it formed the subdominant component $(40.5 \pm 12.5 \%$ and $42.9 \pm 12.3 \%)$ in Bhereki and Holmari beels respectively. The dominance in Ghotonga beel suggested availability of other food resources such as organic matter absorbed in sediments, detritus and bacteria.

Interestingly, this study indicated differences in quantitative importance of zooplankton groups in different beels during the study and also during two successive years. Rotifera $>$ Rhizopoda $>$ Copepoda $>$ Cladocera in Bhereki beel; Rhizopoda $>$ Rotifera $>$ Copepoda $>$ Cladocera in Holmari beel; and Rotifera $>$ Rhizopoda $>$ Cladocera $>$ Copepoda in Ghotonga beel, in the stated order, contributed to zooplankton during the study period. On the other hand, Rotifera $>$ Copepoda $>$ Rhizopoda $>$ Cladocera and Rhizopoda $>$ Rotifera $>$ Copepoda $>$ Cladocera indicated importance in Bhereki beel during two years, respectively. In Holmari beel, Rotifera > Rhizopoda $>$ Copepoda $>$ Cladocera contributed to zooplankton during first year while Rhizopoda $>$ Rotifera $>$ Copepoda $>$ Cladocera showed importance during second year. Further, Rotifera $>$ Rhizopoda $>$ Copepoda $\geq$ Cladocera contributed to zooplankton abundance during first year in Ghotonga beel while Rotifera $>$ Rhizopoda $\geq$ Cladocera $>$ Copepoda deserved mention during second year. The variations are hypothesized to habitat diversity and environmental heterogeneity amongst three beels during the study as well as during two years.

Rotifera, an important group, is characterized by marginal density variations in Bhereki $(80 \pm 22 \mathrm{n} / 1)$, Holmari $(89 \pm 32 \mathrm{n} / \mathrm{l})$ and Ghotonga $(119 \pm 37 \mathrm{n} / \mathrm{l})$ beels with higher mean density during first year in Holmari and during second year in other two beels. It formed the dominant component of zooplankton in Bhereki $(32.8 \pm 6.5 \%)$ and Ghotonga ( $40.4 \pm 6.7 \%$ ) beels while it comprised a sub-dominant group in Holmari beel $(34.0 \pm 10.1 \%)$. The rotifers contributed significantly to zooplankton density variations of Bhereki and Ghotonga $\left(r_{1}=0.697, p\right.$ $\left.=0.0001 ; r_{3}=0.851, p<0.0001\right)$ beels; this generalization is evident from the fact that peak density of Rotifera concurred with zooplankton peak in Ghotonga beel while no such trend was observed in Bhereki beel. ANOVA registered significant rotifer density variations $\left(\mathrm{F}_{2,71}=\right.$ $10.595, \mathrm{P}=0.0001$ ) amongst three beels. The importance of Rotifera in Bhereki and Ghotonga beels agreed with the reports of ${ }^{1,3,6-9,29,35}$ while its sub-dominance in Holmari beel agreed with the reports of. , $28,37,38$ The Rotifera density followed indefinite monthly variations in the sampled beels with peaks during post-monsoon (September, 2010), summer (July, 2011) and winter (January, 2012) in Bhereki, Holmari and Ghotonga beels, respectively. Their post-monsoon peak concurred with the reports from the floodplains of the Kashmir valley, ${ }^{29}$ winter peak concurred with the results from certain floodplain lakes of northeast India ${ }^{5,6,7,8}$ while summer peak concurred with the reports of. ${ }^{35,37}$ Lecanidae > Lepadellidae contributed notably to Rotifera abundance in Bhereki and Holmari beels while Lecanidae $>$ Brachionidae contributed in Ghotonga beel. The importance of the littoral periphytonic taxa of three Eurotatorien families is attributed to lack of true limnetic conditions in the sampled beels. The lack of dominance of individual rotifer species in any of the sampled lakes suggested that the rotifers are generalists in terms of general environment. ${ }^{3}$

Rhizopoda, a dominant group of zooplankton of Holmari beel $(34.3 \pm 11.6 \%)$ and a sub-dominant component $(29.5 \pm 11.0 \%$, $26.4 \pm 7.5 \%$ ) in Bhereki and Ghotonga beels, registered insignificant variations amongst the sampled beels. The rhizopods contributed significantly to zooplankton density only in Holmari beel $\left(r_{2}=\right.$
$0.846, \mathrm{p}<0.0001)$; this generalization is supported by the fact that their maxima contributed to zooplankton peak in this wetland. This group followed no definite pattern of quantitative variations during the study period and recorded peaks during monsoon (August, 2012), pre-monsoon (April, 2012) and autumn (September, 2010) in Bhereki, Holmari and Ghotonga beels, respectively. The present results differed from summer periodicity of these testaceans vide. ${ }^{5,39}$ Arcellidae > Centropyxidae $>$ Difflugidae contributed to the Rhizopoda abundance in Bhereki and Ghotonga beels, respectively while Arcellidae > Centropyxidae $>$ Euglephidae contributed to their density in Holmari beel. Arcella discoides and A. vulgaris collectively influenced the rhizopod abundance in Bhereki beel; Arcella discoides, A. vulgaris, Centropyxis aculeata, Euglypha acanthophora and Nebela caudata showed importance in Holmari beel; while Arcella discoides, A. vulgaris and Centropyxis aculeata deserved mention in Ghotonga beel.

Copepoda is a sub-dominant group of Bhereki $>$ Holmari $>$ Ghotonga beels; the stated role was in contrast to their dominance reported by. ${ }^{5,28,37}$ It indicated significant annual $\left(\mathrm{F}_{1,23}=21.832, \mathrm{P}=\right.$ $0.0006)$ as well as significant monthly $\left(\mathrm{F}_{11.23}=4.073, \mathrm{P}=0.014\right)$ variations in Bhereki beel. This group registered no definite pattern of monthly density variations during the study in the sampled beels and registered peak values during autumn in Bhereki (November, 2010) and Holmari (October, 2010) beels and during early summer (April, 2011) in Ghotonga beel. Cyclopoids mainly influenced quantitative variations of this group in Bhereki, Holmari and Ghotonga beels, respectively; this reflected the prevalence of stable environmental conditions for these ' $\mathrm{k}$-strategists'. ${ }^{40,41}$ Tropocyclops prasinus showed importance in the three beels, respectively; Mesocyclops leuckarti showed certain importance in Holmari $>$ Ghotonga beels while Thermocyclops decipiens deserved mention in Bhereki beel and Microcyclops varicans indicated limited role in Holmari beel. The occurrence of nauplii throughout the study showed an active continuous reproductive phase of the cyclopoids. ${ }^{6,8,9,42}$

Cladocera formed another sub-dominant group in Ghotonga $>$ Bhereki $>$ Holmari beels respectively, and registered significant density variations amongst three beels $\left(\mathrm{F}_{2.71}=5.872, \mathrm{P}=0.005\right)$. The Cladoceran abundance followed no definite pattern of monthly density variations in the sampled beels and registered peak values during autumn (October, 2010) in Bhereki beel and during winter (January, 2011 and January, 2012) in Holmari and Ghotonga beels, respectively. The winter peaks concurred with the reports of Sharma. ${ }^{6,9}$ The Cladocera were characterized by importance of Chydoridae in all three beels concurrent with the results of:, ${ }^{1,-9}$ Daphniidae and Macrothricidae were other important families in the sampled beels while Macrothrix triserialis showed certain value in Ghotonga beel. Ostracoda, another group of zooplankton, indicated very poor abundance in the sampled beels.

Zooplankton of the Majuli beels are characterized by consistently high species diversity throughout the study with higher diversity ( $>$ 4.0) during May, 2011 (summer), November, 2011 (autumn) and August, 2012 (monsoon) in Ghotonga beel and during August, 2012 (monsoon) in Holmari beel. The interesting feature is hypothesized to habitat diversity and environmental heterogeneity of the sampled beels. High diversity with lower densities of majority of species in different beels is attributed to fine niche portioning amongst zooplankton species in combination with micro- and macro-scale habitat heterogeneity as hypothesized by Segers $\mathrm{H}^{43}$ and affirmed by. ${ }^{6-}$ ${ }^{9}$ This generalization is endorsed by relative quantitative importance of only ten out of a total of 141 zooplankton species known from the sampled beels with only three namely Arcella discoides, A 
vulgaris and Tropocyclops prasinus common to all three beels but in relatively low average densities. The low densities of the rest of species, suggested that the majority of zooplankton is generalists in terms of general environment as hypothesized by Sharma BK. ${ }^{3}$ ANOVA showed significant diversity variations amongst three beels $\left(\mathrm{F}_{2.23}=13.046, \mathrm{P}=3.25 \mathrm{E}-05\right.$. The present study did not follow any definite annual and monthly patterns of zooplankton diversity in the three sampled beels.

Lower zooplankton dominance of Bhereki, Holmari and Ghotonga beels is attributed to lack of distinct quantitative importance of different species coupled with low densities of majority of species. The former is hypothesized ${ }^{44}$ to the fact that the habitat of the sampled Majuli beels had resources for utilization by majority of species and thus providing high amount of niche overlap. This generalization holds valid throughout the study except of limited role of Arcella discoides, A. vulgaris, Tropocyclops prasinus in fewer collections in the three beels; it is affirmed by inverse correlations between dominance vs. diversity in Bhereki $\left(r_{1}=-0.700 \mathrm{p}=0.0001\right)$, Holmari $\left(\mathrm{r}_{2}=-0.880, \mathrm{p}\right.$ $<0.0001)$ and Ghotonga $\left(r_{3}=-0.799, p<0.0001\right)$ beels, respectively. High evenness affirmed low densities and equitable abundance of various species and reiterated that the majority of zooplankton are 'generalists' vis-à-vis their general environment. ${ }^{3}$ ANOVA registered significant dominance $\left(\mathrm{F}_{2,71}=8.009, \mathrm{P}=0.001\right)$ and evenness variations $\left(\mathrm{F}_{2,71}=5.070, \mathrm{P}=0.010\right)$ amongst three beels.

\section{Limnological correlations}

This study indicated insignificant influence of individual abiotic parameters on zooplankton richness. Of the different groups, only Rotifera richness is positively correlated with dissolved organic matter $\left(r_{2}=0.551, p=0.0026\right)$ in Holmari beel. Zooplankton abundance is inversely correlated with specific conductivity $\left(r_{3}=-0.598, p=0.002\right)$ in Ghotonga beel; Rhizopoda abundance is inversely correlated with water temperature $\left(r_{2}=-0.556, p=0.0024\right)$ in Holmari beel and directly correlated with $\mathrm{pH}\left(\mathrm{r}_{3}=0.567, \mathrm{p}=0.0019\right)$ in Ghotonga beel; Cladocera positively correlated with sulphate $\left(\mathrm{r}_{3}=0.565, \mathrm{p}=\right.$ 0.002 ) in Ghotonga beel while Copepoda positively correlated with total hardness $\left(r_{1}=0.565, p=0.002\right)$ and magnesium $\left(r_{1}=0.555\right.$, $\mathrm{p}=0.0024)$ in Bhereki beel and is negatively correlated with total dissolved solids $\left(r_{3}=-0.540, p=0.0032\right)$ in Ghotonga beel. Rotifera abundance exhibited no significant correlation of any abiotic parameter in the sampled beels. The results thus concluded limited influence of abiotic factors on richness and abundance of zooplankton in this study.

Canonical correspondence analysis (CCA) with 17 abiotic factors recorded lower cumulative influence on zooplankton assemblages along first two axes of $61.33 \%, 58.18 \%$ and $63.77 \%$ in Bhereki, Holmari and Ghotonga beels, respectively. The results showed the importance of water temperature, $\mathrm{pH}$, specific conductivity, hardness, magnesium, dissolved organic matter, total dissolved solids and nitrate for zooplankton taxa in Bhereki beel. Water temperature, free carbondioxide, specific conductivity, dissolved organic matter, hardness, chloride and silicate reflected importance in Holmari beel while water temperature, $\mathrm{pH}$, magnesium, hardness, dissolved organic matter, total dissolved solids and sulphate showed importance in Ghotonga beel.

While explaining limited influence of individual abiotic and lower cumulative influence of 17 abiotic variables on zooplankton assemblages (vide Canonical Correspondence Analysis), this study suggested that zooplankton taxa are rather generalists in terms of general abiotic factors, with factors associated with microhabitat being more important. The latter feature supported hypothesis of
Sharma and Sharma ${ }^{3}$ vis-a-vis abiotic factors on Rotifera diversity in the floodplain lakes of northeast India.

To sum up, this study merits ecosystem diversity importance vis-à-vis quantitative dominance of the species rich zooplankton of Ghotonga beel and its sub-dominance of Bhereki and Holmari beels with quantitative importance of Rotifera $>$ Rhizopoda in Bhereki and Ghotonga beels, and of Rhizopoda > Rotifera in Holmari beel. The richness, abundance and species diversity of zooplankton followed no definite pattern of monthly variations. The results affirmed higher species diversity, higher evenness and lower dominance of zooplankton and are characterized by lower densities of a majority of species. The limited individual influence and low cumulative influence (vide CCA) of seventeen abiotic factors on zooplankton assemblages of the Majuli beels affirmed that zooplankton taxa are rather generalists in terms of general abiotic factors and thus suggested importance of factors associated with microhabitat variations.

\section{Acknowledgements}

We thank the Head, Department of Zoology, NEHU, Shillong, for laboratory facilities. The field-work for this study was partially supported under the 'UPE (Biosciences) Program' of North-Eastern Hill University, Shillong.

\section{Conflicts of Interest}

The authors have no conflict of interests.

\section{References}

1. Sharma S, Sharma BK Zooplankton diversity in floodplain lakes of Assam, India. Records of the Zoological Survey of India, Occasional Paper No, 2008;290:1-307.

2. Sharma S, Sharma BK Faunal Diversity of Aquatic Invertebrates of Deepor Beel (a Ramsar site), Assam, northeast India. Wetland Ecosystem Series. 2013;17:1-227.

3. Sharma BK, Sharma S Northeast India-An important region with a rich biodiversity of Rotifera. In: Sharma BK, et al. (Eds.), Rotifera XIII: Rotifer Biology - A structural and functional Approach. International Review of Hydrobiology. 2014;99(1-2):20-37.

4. Sugunan VV Fisheries management of small bodies in seven countries in Africa, Asia and Latin America. FAO Circular No. 1997;933:1-149.

5. Sharma BK, Hussain Abundance and ecology of zooplankton in a tropical floodplain lake, Assam (N.E. India). Ecology, Environment and Conservation. 2001;7(4):397-403.

6. Sharma BK Zooplankton communities of Deepor beel (a Ramsar site), Assam (N. E. India): Ecology, Richness and Abundance. Tropical Ecology. 2011;52(3):291-302.

7. Sharma BK, Sharma S Diversity of zooplankton of a tropical floodplain lake of the Brahmaputra river basin, Assam (Northeast India). Opuscula Zoologica, Budapest. 2012;43(2):187-195.

8. Sharma BK Zooplankton diversity of two floodplain lakes (pats) of Manipur, northeast India. Opuscula Zoologica, Budapest. 2011;42(2):185-197.

9. Sharma BK, Sharma S Zooplankton diversity of Loktak Lake, Manipur, India. Journal of Threatened Taxa. 2011;3(5):1745-1755.

10. Sharma BK Rotifers (Rotifera: Eurotatoria) from wetlands of Majuli the largest river island, the Brahmaputra river basin of upper Assam, northeast India. Check List. 2014;10(2):292-298.

11. Sharma BK, Sharma S, Hatimuria MK Rotifer assemblages (Rotifera: Eurotatoria) of the floodplain lakes of Majuli River Island, the Brahmaputra river basin, northeast India. International Journal of Aquatic Biology. 2015;3(1):1-13. 
12. Sharma BK, Hatimuria MK, Sharma S Ecosystem diversity of Cladocera (Crustacea: Branchiopoda) of the floodplain lakes of Majuli River Island, the Brahmaputra river basin, northeast India. International Journal of Aquatic Biology. 2015;3(2):78-88.

13. APHA Standard Methods for the Examination of Water and Wastewater. (18 ${ }^{\text {th }}$ edn), American Public Health Association, Washington, USA. 1992

14. Koste W ROTATORIA. Die Radertiere Mitteleuropas, begrundet von Max Voigt. Uberordnung Monogononta. Gebruder Borntaeger, Berlin, Stuttgart. I. Text, pp. 1978;673.

15. Michael RG, Sharma BK Indian Cladocera (Crustacea: Branchiopoda: Cladocera) Fauna of India and adjacent countries Series. Published by Zoological Survey of India, India, pp. 1988;262.

16. Sharma BK Freshwater rotifers (Rotifera: Eurotatoria). Fauna of West Bengal State Fauna Series. 1998;3:341-461. Published by Zoological Survey of India, India.

17. Sharma BK, Sharma S Freshwater Rotifers (Rotifera: Eurotatoria) In: Fauna of Meghalaya: State Fauna Series, 1999a;4(9):11-161. Published by Zoological Survey of India, India.

18. Sharma BK, Sharma S Freshwater Cladocerans (Crustacea: Branchiopoda: Cladocera). In: Fauna of Meghalaya: State Fauna Series. 1999b;4(9):469-550. Published by Zoological Survey of India, India.

19. Sharma BK, Sharma S (2000) Freshwater Rotifers (Rotifera: Eurotatoria). State Fauna Series: Fauna of Tripura 7: 163-224. Published by Zoological Survey of India, India.

20. Ludwig JA, Reynolds JF Statistical ecology: a primer on methods and computing. John Wiley and Sons, USA, pp. 1988;337.

21. Magurran AE Ecological diversity and its measurement. Croom Helm Limited, London. 1988;179 pp.

22. Talling JF, Talling IB The chemical composition of African lake waters Internationale Revue gesammten Hydrobiologie. 1965;50:421-463.

23. Wanganeo A, Wanganeo S Plankton Diversity in Kashmir Himalayan Lakes: An Overview. Trends in Biodiversity \& Aquaculture. Daya Publishing House, India, pp. 2006a;130-160.

24. Wanganeo A, Wanganeo S Variation in zooplankton population in two morphometrically dissimilar rural lakes of Kashmir Himalayas. Proceedings of the National Academy of Sciences, India. 2006b;76(B):222-239.

25. Sharma PC, Pant MC Structure of a littoral zooplankton community of two Kumaun lakes, (U.P.), India. Limnologica (Berlin). 1984;16 (1):5165 .

26. Sharma PC, Pant MC Species composition of zooplankton in two Kumaun Himalayan lakes (UP, India). Archiv für Hydrobiologie. $1985 ; 102: 387-403$

27. Datta T Zooplankton diversity and physicochemical conditions of two wetlands of Jalpaiguri district, India. International journal of Applied Biology and Pharmaceutical Technology. 2011;2(3):576-583.

28. Khan RA Faunal diversity of zooplankton in freshwater wetlands of South-eastern West Bengal. Records of the Zoological Survey of India, Occasional Paper No. 2003;204:1-107.
29. Khan MA Observations on zooplankton composition, abundance and periodicity in two flood-plain lakes of the Kashmir-Himalayan valley. Acta hydrochemica Hydrobiolologica. 1987;15(2):167-174.

30. Slathia D, Dutta SPS Hydrobiological study of a subtropical Shiwalik Lake, Jammu, J\&K (India). International Journal of Chemical, Environmental \& Biological Sciences. 2013;1(1):2320-4087.

31. Zutshi DP, Vass KK. Limnological Studies on Dal Lake, Srinagar. III. Biological Features. Proceedings of the Indian National Science Academy, section B. 1982;48(2):234-241.

32. Mir AR, Wanganeo A, Yousuf AR, et al. Zooplankton community in a through flow system of Kashmir Himalayan wetland. Nature Environment and Pollution Technology. 2008;7(4):627-634.

33. Ganesan L, Khan RA. Studies on the Ecology of Zooplankton in a Floodplain Wetland of West Bengal, India. In Sengupta M, Dalwani R (Eds.), Proceedings of Taal 2007: The 12th World Lake Conference. 2008:67-73.

34. Rai DN, Dutta Munshi JM. Ecological characteristics of "chaurs" of North Bihar. In. Gopal B, et al. (Eds.), Wetlands: Ecology and Management. Vol. 11, International Scientific Publications \& National Institute of Ecology, India. 1988;p.89-95.

35. Sanjer L R, Sharma UP. Community structure of plankton in Kawar lake wetland, Begusarai, Bihar: II Zooplankton. Journal of Freshwater Biology. 1995;7:165-167.

36. Vass KK. Beel fisheries resources in West Bengal. Bulletin of Central Inland Capture Fisheries Research Institute, Barrackpore. 1989;63:29 35 .

37. Baruah A, Sinha AK, Sharma UP. Plankton variability of a tropical wetland, Kawar (Begusarai), Bihar. Journal of Freshwater Biology. $1993 ; 5: 27-32$

38. Sharma BK. Synecology of Rotifers in a tropical floodplain lake of Upper Assam (N. E. India). Indian Journal of Animal Sciences. 2000;70:880-885.

39. Sinha AK, Baruah A, Singh DK, et al. Biodiversity and pollution status in relation to physico-chemical factors of Kawar lake (Begusarai), North Bihar. Journal of Freshwater Biology. 1994;6:309-331.

40. Allen JD. Life history patterns in zooplankton. Am. Nat. 1976;110:165180 .

41. Schmidt-Araya JM, Zuniga AR. Zooplankton community structure in two Chilean reservoirs. Archiv für Hydrobiologie. 1992;123(3):305-335.

42. Sharma BK, Pachuau L. Zooplankton diversity of a sub-tropical reservoir of Mizoram, Northeast India. Opuscula Zoologica Budapest. 2013;44(1):47-60.

43. Segers H. Global diversity of rotifers (Rotifera) in freshwater. Hydrobiologia. 2008;595:49-59.

44. Mac Arthur RH. Patterns of spices diversity. Biological Reviews. 1965;40(4):510-533. 